\title{
Lipid and protein oxidation in female patients with chronic fatigue syndrome
}

Slavica Tomic ${ }^{1}$, Snezana Brkic ${ }^{1}$, Daniela Maric ${ }^{1}$, Aleksandra Novakov Mikic ${ }^{2}$

${ }^{1}$ Clinic for Infectious Diseases, Clinical Center Vojvodina, Novi Sad, Serbia ${ }^{2}$ Clinic for Gynecology and Obstetrics, Clinical Center Vojvodina, Novi Sad, Serbia

Submitted: 17 November 2010

Accepted: 4 April 2011

Arch Med Sci 2012; 8, 5: 886-891

DOI: 10.5114/aoms.2012.31620

Copyright (C 2012 Termedia \& Banach

\section{Corresponding author:}

Daniela Maric MD

Clinical Center Vojvodina

Clinic for Infectious Diseases

Hajduk Veljkova 1-11

21000 Novi Sad, Serbia

Phone: +381641526022

E-mail: dijetinfo@gmail.com

\begin{abstract}
Introduction: Chronic fatigue syndrome (CFS) is a widely recognized problem, characterized by prolonged, debilitating fatigue and a characteristic group of accompanying symptoms, that occurs four times more frequently in women than in men. The aim of the study was to determine the existence of oxidative stress and its possible consequences in female patients with CFS.

Material and methods: Twenty-four women aged 15-45 who fulfilled the diagnostic criteria for CFS with no comorbidities were recruited and were age matched to a control group of 19 healthy women. After conducting the routine laboratory tests, levels of the lipid oxidation product malondialdehyde (MDA) and protein oxidation protein carbonyl (CO) were determined.

Results: The CFS group had higher levels of triglycerides $(p=0.03), \operatorname{MDA}(p=0.03)$ and $\mathrm{CO}(p=0.002)$ and lower levels of HDL cholesterol $(p=0.001)$ than the control group. There were no significant differences in the levels of total protein, total cholesterol or LDL cholesterol.

Conclusions: The CFS group had an unfavorable lipid profile and signs of oxidative stress induced damage to lipids and proteins. These results might be indicative of early proatherogenic processes in this group of patients who are otherwise at low risk for atherosclerosis. Antioxidant treatment and life style changes are indicated for women with CFS, as well as closer observation in order to assess the degree of atherosclerosis.
\end{abstract}

Key words: chronic fatigue syndrome, oxidative stress, malondialdehyde, protein carbonyl, atherosclerosis.

\section{Introduction}

Chronic fatigue syndrome is defined as fatigue lasting more than 6 months, associated with secondary symptoms, and inducing a 50\% decreased ability to participate in ordinary activities [1]. It was first defined in 1988 by the US Center for Disease Control and Prevention (CDC), but the definition was later revised by Fukuda et al. by excluding any active or suspected medical or psychiatric affection and substance abuse as well as severe obesity as defined by a body mass index equal to or greater than $45 \mathrm{~kg} / \mathrm{m}^{2}$ [2]. The disorder is now a widely recognized problem and the diagnosis can be made after alternative medical and psychiatric causes of chronic fatiguing illness have been excluded [3]. Although there is still lack of a specific laboratory test or marker to identify it, chronic fatigue syndrome (CFS) should be considered when a patient presents with prolonged fatigue lasting 6 or more months (major criteria) and with at least 
four of the following symptoms (minor criteria): selfreported impairment in short-term memory or concentration difficulties, sore throat, tender cervical or auxiliary lymph nodes, muscle pain, multi-joint pain without joint swelling or redness, headaches of a new type, pattern, or severity, unrefreshing sleep, and post-exertional malaise lasting more than $24 \mathrm{~h}[1]$.

In the primary care setting, the prevalence of CFS ranges from $3 \%$ to $20 \%$ depending on the diagnostic criteria used [4]. People of every age, gender, ethnicity and socioeconomic group can have CFS but it affects women at six times the rate of men [5]. Moreover, it seems that the symptoms of CFS are more severe in women: in the Chicago community-based sample, gender predicted fatigue severity, with women exhibiting higher fatigue scores than men. Also, within this sample women had significantly poorer physical functioning, more bodily pain, poorer emotional functioning, significantly more severe muscle pain, and significantly greater impairment of work activities [6, 7]. More than $80 \%$ of CFS sufferers go undiagnosed [8].

Despite the controversy surrounding CFS, there are numerous studies on the pathogenesis and treatment options for this condition [9]. Viral triggers and immune dysregulation, neuroendocrine or central nervous system dysfunction and muscular function disturbances have all been considered as pathophysiological factors [3, 5, 9-13]. There is mounting evidence that oxidative stress plays an important part in the pathogenesis of CFS by affecting different systems and tissues [14-21]. Moreover, the level of oxidative stress correlates with the severity of symptoms [19]. Investigations of the type and severity of oxidative stress in CFS have mostly dealt with lipid peroxidation (LPO) [17, $19,28]$. One of the most important LPO products is malondialdehyde (MDA), which serves as a potential biomarker of oxidative damage and disease severity [22-29].

Oxidative attack on proteins results in the formation of protein carbonyls (CO), often with the loss of functionality of the parent protein. To our knowledge, there has been only one study concerning protein oxidation and CFS, which showed a significant elevation of protein carbonyl levels in sera of CFS [30]. Protein carbonyls (CO) are reported to be the most frequently studied marker of oxidative damage to proteins [31] and the use of protein CO groups as biomarkers of oxidative stress has some advantages in comparison with the measurement of other oxidation products because of the relative early formation and stability of carbonylated proteins [32, 33].

The aim of this study was to determine the existence of oxidative stress and its possible consequences in female patients affected with CFS.

\section{Material and methods}

\section{Study population}

Consecutive patients who had been referred to the University Clinic for Infectious Diseases with a complaint of fatigue lasting for more than 6 months were considered for the study. As CFS is more frequent in female patients and the symptoms are more pronounced in this group, only females of generative age (15-45) were considered for the study. Also, the aim was to form a homogeneous group of patients regarding level of activity, body composition and general health status in order to limit influences on oxidative stress levels.

Patients were included in the study if they met the 1994 CDC definition: two major criteria (disabling fatigue lasting more than 6 months and exclusion of all other potential causes of fatigue) and at least four minor criteria (fever, sore throat, lymphadenopathy, muscle weakness, myalgia, headache, sleep disturbances and neuropsychological complaints).

Patients with the following conditions were also excluded from the study: acute severe illness during the previous 6 months, hypo or hypertension, pregnancy, chronic liver disease, thyroid dysfunction, suprarenal dysfunction, hypertension, diabetes mellitus, obesity (body mass index (BMI) greater than $30 \mathrm{~kg} / \mathrm{m}^{2}$ ), autoimmune disorders, a history of malignancy, alcohol or drug abuse and the presence of any psychiatric disorders. Also, patients who fulfilled the CFS criteria but had received any kind of medication for the condition were excluded from the study.

The control group comprised female subjects who had no medical complaints and had volunteered to participate in the study. They underwent the same medical evaluation process as the CFS group and were confirmed to be healthy. The control group was matched to the CFS group in terms of age and smoking habits.

The study was approved by the relevant ethical committees and all patients signed informed consent before entering the study.

The subjects entering the study underwent a complex medical evaluation. This included detailed history of the present illness, past medical history and a physical examination. Height and weight measurements were recorded for all subjects and BMI was calculated.

The levels of CFS symptoms were estimated using the Fibro Fatigues Scale (FFS), which is a self assessment 16-item questionnaire. Fibro Fatigues Scale has been used previously in numerous studies and has proved to be a useful and valid scale for assessing the degree of the symptoms in CFS in clinical studies [34-36]. 
After the examination, routine laboratory tests were performed. These included a complete blood count, erythrocyte sedimentation rate, $C$ reactive protein, tests for autoantibodies, thyroid, kidney, suprarenal and liver function tests, as well as levels of total cholesterol (TC), triglycerides (TG), LDL cholesterol (LDL-C) and HDL cholesterol (HDL-C). Also, ELISA tests were performed to exclude the most important acute viral and bacterial infections that can influence oxidant status (Coxsackie B, adenovirus, EBV, CMV, HSV, HIV, HBV, HCV and Borrelia burgdorferi).

\section{Oxidative stress markers}

After inclusion in the study, $5 \mathrm{ml}$ of venous blood was obtained from each patient and sera were immediately centrifuged and then refrigerated at $-20^{\circ} \mathrm{C}$.

Levels of MDA were measured using the OxiSelect MDA Adduct ELISA kit, one of the first commercial ELISA tests for research use only. The manufacturer revised protocol was followed. BSA standards or protein samples $(10 \mu \mathrm{g} / \mathrm{ml})$ were adsorbed onto a 96 -well plate for $2 \mathrm{~h}$ at $37^{\circ} \mathrm{C}$. The MDA-protein adducts present in the sample or standard were probed with an anti-MDA antibody, followed by an HRP conjugated secondary antibody. The MDA protein adducts content in an unknown sample was determined by comparing with a standard curve that was prepared from predetermined MDA-BSA standards. Sensitivity of the method varies from $1,875 \mathrm{pmol} / \mathrm{mg}$ to $120 \mathrm{pmol} / \mathrm{mg}$ MDA adduct.
Levels of CO were measured using the OxiSelect Protein Carbonyl ELISA kit, by Cell Biolabs. This test was developed for rapid detection and quantization of protein carbonyl. The quantity of protein carbonyl in protein samples was determined by comparing the absorbance with that of a known reduced/oxidized BSA standard curve. The sensitivity of the test ranges from $0.375 \mathrm{nmol} / \mathrm{mg}$ to $7.5 \mathrm{nmol} / \mathrm{mg}$.

\section{Statistical analysis}

Data were analyzed using SPSS 8.0 software. Differences between the 2 groups were assessed by $\chi^{2}$ tests, the Mann-Whitney test and ANOVA for several groups. The relationship between the various parameters was assessed by correlation. Data were expressed as mean (95\% confidence interval for mean) and two-tailed $p$-values less than 0.05 were considered statistically significant.

\section{Results}

Twenty-four women who fulfilled the CFS criteria and 19 healthy women, aged 15-45 years, were included in the study. According to the design of the study the two groups, i.e. the CFS group and the control group, were similar in age and smoking habits. Subjects in both groups were nonobese and the difference in BMI between the two groups was not significant (Table I). ANOVA statistical analysis showed a significant difference between groups in all 12 questionnaire items ( $p<0.0001)$. The median of the total FFS score was higher in the CFS group than in the control group $(X=21.90$,

Table I. Lipid profile and oxidative stress markers in patients with CFS and in healthy controls

\begin{tabular}{|c|c|c|c|}
\hline Variable & $\begin{array}{c}\text { CFS group }(n=24) \\
\text { Mean (min.-max.; SD) }\end{array}$ & $\begin{array}{l}\text { Control group }(n=19) \\
\text { Mean (min.-max.; SD) }\end{array}$ & Value of $p$ \\
\hline Age & $33.33(24-47 ; 5.65)$ & $33.37(25-44 ; 5.28)$ & 0.9 \\
\hline Smokers: & 9 & 7 & 0.8 \\
\hline Heavy & 4 & 3 & 1.0 \\
\hline Light & 5 & 4 & 1.0 \\
\hline $\mathrm{BMI}\left[\mathrm{kg} / \mathrm{m}^{2}\right]$ & $22(19-25 ; 0.3)$ & $24(18-28 ; 0.4)$ & 0.9 \\
\hline C-reactive protein [mg/l] & $1.05(1-8.2 ; 1.4)$ & $1.2(0-21 ; 3.3)$ & 1.0 \\
\hline Fibrinogen $[\mathrm{g} / \mathrm{l}]$ & $3.1(1.8-5.3 ; 0.67)$ & $3.4(2.2-6.2 ; 0.9)$ & 0.9 \\
\hline Glucose in blood [mmol/l] & $4.5(3.1-5.8 ; 0.8)$ & $4.8(3.2-5.6 ; 0.6)$ & 0.9 \\
\hline Triglycerides [mmol/l] & $1.038(0.5-2.0 ; 0.39)$ & $0.798(0.5-1.4 ; 0.27)$ & 0.03 \\
\hline Total cholesterol [mmol/l] & $4.636(3.0-6.7 ; 1.1)$ & 4.895 (3.9-6.7; 0.81) & 0.2 \\
\hline LDL cholesterol [mmol/l] & $3.253(2.2-4.7 ; 0.83)$ & $3.024(0.3-4.7 ; 0.91)$ & 0.6 \\
\hline HDL cholesterol [mmol/l] & $1.181(0.9-1.6 ; 0.23)$ & $1.474(0.9-1.9 ; 0.28)$ & 0.001 \\
\hline MDA [pmol/mg] & $15.05(0.0-30.1 ; 9.12)$ & $10.13(0.0-22.4 ; 8.92)$ & 0.03 \\
\hline Protein carbonyl [nmol/mg] & $3.18(0.3-5.9 ; 1.41)$ & $1.78(0-4.2 ; 1.09)$ & 0.002 \\
\hline
\end{tabular}

*To convert mmol/l of blood glucose to $\mathrm{mg} / \mathrm{dl}$, multiply by 18 ; to convert mmol/l of total cholesterol, HDL or LDL cholesterol to mg/dl, multiply by 39; to convert mmol/l of triglycerides to $\mathrm{mg} / \mathrm{dl}$, multiply by 89 
$\mathrm{SD}=5.23$ vs. $X=2.93, \mathrm{SD}=1.87, p<0.0001)$, as expected.

There were no significant differences in the routine laboratory tests (complete blood count; erythrocyte sedimentation rate; tests for autoantibodies; and tests of thyroid, kidney, suprarenal and liver function). The results of all tests were within the normal range for both groups, which was expected as patients with co-morbidities were not included in the study.

C-reactive protein, fibrinogen and blood glucose levels were also in the normal range and did not differ between the groups (Table I).

However, in the case of the levels of TG and $\mathrm{HDL}-\mathrm{C}$, there was a difference between groups (Table I). The CFS group had higher levels of TG and lower levels of HDL-C. No such difference was found in the levels of TC and LDL-C. The MDA levels were significantly higher in the CFS group. Levels of CO were also higher in the CFS group than in the control group. The FFS score was correlated with both MDA and CO levels and no significant correspondences were found.

\section{Discussion}

In this study we found increased levels of both MDA and CO levels in the CFS group. We can therefore conclude that oxidative stress is present in our group of female CFS patients.

Although there are still many controversies concerning CFS, it is now a well-established condition with several million sufferers. It is likely that physicians avoid making this diagnosis due to the fact that CFS is still a diagnosis per exclusionem and that it has well-known overlapping syndromes. There is also the fact that many CFS patients do not seek medical attention due to their social and/or economic status. Nevertheless, many scientists are investigating the answers to the numerous questions CFS still poses: epidemiology, etiology, pathogenesis and treatment have not been completely defined.

Among the possible mechanisms of pathogenesis, oxidative stress is especially interesting as it can lead to potential therapeutic options and secondary prevention. It appears to play an important role in the development of, and also contributes to, some of the symptoms in CFS [14-21].

The MDA is a product of lipid peroxidation of polyunsaturated fatty acids and it can be found in physiological conditions in lower concentrations. High levels of MDA are indicative of oxidative stress. The MDA is potentially very noxious [22] and its toxicity can be directed towards cardiovascular stability. Primarily, MDA reacts with apoB fractions of oxidized lipoproteins (LDL), which causes impaired interaction of the modified lipoproteins and macrophages [22, 37]. This phenomenon is the basis of atherogenicity. Another probable toxic action of MDA involves collagen. Even if the nature of the cross-link has not yet been determined in detail, the inter-molecular cross-linking of collagen through MDA may significantly contribute to the stiffening of cardiovascular tissue [22]. It is quite possible that the presence of MDA at higher levels may predict the insurgence of vascular pathologies. Polidori and collaborators found higher levels of antioxidants and lower levels of MDA in healthy subjects when compared in a case-control study with congestive heart failure patients [23]. Plasma from atherosclerotic patients was richer in MDA studied by Tamer et al. [38]. In two studies reported by Boaz et al. [39, 40], serum MDA values were markedly higher in hemodialysis patients with cardiovascular complications than in those without such complications.

The second significant finding in the CFS group was raised levels of $\mathrm{CO}$, the product of protein oxidation. As generation of carbonyl derivatives occurs by many different mechanisms, the level of CO groups in proteins is widely used as a marker of oxidative protein damage, often correlating well with the progression of the disease. To date, accumulation of $\mathrm{CO}$ has been observed in several human diseases including Alzheimer's, Parkinson's and Huntington's diseases; diabetes; inflammatory bowel disease; adult (or acute) respiratory distress syndrome; chronic renal and lung diseases; sepsis; arthritis; preeclampsia and amyotrophic lateral sclerosis [31, 41, 42]. The level of oxidized proteins also increases with aging and in age-related diseases [31-33], which is why it was important that the two groups studied were matched in terms of age. Protein oxidation products in CFS patients have so far been investigated only by Smirnova and Pall [30]. This group had similar results to ours but they found even more significantly elevated CO levels $(p<0.0005)$ in CFS patients compared to controls.

The third important finding of our study was the unfavorable lipoprotein profiles in the CFS group. We found significantly lower levels of HDL-C and higher levels of TG in the study group. HDL-C is the beneficial form of blood cholesterol which protects the artery wall from atherosclerosis [43]. Even in individuals whose LDL levels are low, HDL remains a strong independent predictor of coronary artery disease risk [44]. Besides preventing cholesterol accumulation in cells of the artery wall, HDL-C acts as an anti-inflammatory agent by degrading lipid oxidation products [43-47]. In this way, HDL-C acts against products of lipid peroxidation which are potentially atherogenic. We also found higher TG levels in the CFS group of patients. High levels of triglycerides are a well-known factor for atherosclerosis and are included in the Framingham charts 
that have been widely used by clinicians to quantify an individual's absolute risk for coronary heart disease.

Oxidative stress plays an important role in atherosclerosis. The LDL carries cholesterol from the liver to the circulatory system. It is susceptible to oxidation by reactive oxygen species (ROS), and damage is seen both to the lipid and to the protein moiety $[48,49]$. In an environment where oxidative stress is present and where there are lower levels of antioxidant HDL-C and higher levels of TG, oxidation of LDL might be more pronounced [50].

To date, only a few studies have been conducted that have implicated an increased risk of cardiovascular events in CFS patients with no comorbidities. Kennedy et al. used 8-Iso-prostaglandin $\mathrm{F}_{2 \alpha}$ as a lipid peroxidation marker with a potential potent biological activity [19]. They found significantly elevated levels of F2-isoprostanes and oxidized LDL and decreased HDL in nonobese, normotensive patients. Isoprostane levels were even greater in CFS patients who had additional cardiovascular risk factors. Similarly to our study, they concluded that CFS patients have a lipid profile and oxidant biology that is consistent with cardiovascular risk.

Three years later, the same group observed a relationship between inflammation and oxidative stress and augmentation index, a measure of arterial stiffness [51]. They have shown that patients with CFS have higher serum CRP levels, elevated levels of isoprostanes and oxLDL, and significantly increased Alx@75, which indicates a significantly increased risk of a future cardiovascular event in these patients.

We also suggest a follow-up of our group of patients which should include an imaging technique in order to assess possible early atherosclerosis.

One limitation of the present study is that we did not measure the levels of oxidized LDL, which is associated with the development of atherosclerosis. A second limitation is the small number of patients. This is mainly due to difficulties in recruiting patients due to the relatively low incidence and prevalence of CFS. Furthermore, we were faced with skepticism about CFS both from the patients and the medical community, which made it difficult to mobilize patients.

In conclusion, in the present study we used two reliable biomarkers of oxidative stress rarely used before with CFS patients. We found signs of lipid and protein oxidative damage, as well as an unfavorable lipid profile in nonobese premenopausal female CFS patients with no comorbidities. We suggest that in this otherwise low risk group there are signs of possible early atherogenesis. This would mean that women who have CFS are at an increased risk for cardiovascular events and are in need of early intervention including antioxidant supplementation and LDL lowering strategies.

\section{References}

1. Holmes GP, Kaplan JE, Gantz NM, et al. Chronic fatigue syndrome: a working case definition. Ann Intern Med 1988; 108: 387-9.

2. Fukuda K, Straus SE, Hickie I, Sharpe MC, Dobbins JG, Komaroff A. The chronic fatigue syndrome: a comprehensive approach to its definition and study. International Chronic Fatigue Syndrome Study Group. Ann Intern Med 1994; 121: 953-9.

3. Griffith JP, Zarrouf FA. A systematic review of chronic fatigue syndrome: don't assume it's depression. Prim Care Companion J Clin Psychiatry 2008; 10: 120-8.

4. Centers for Disease Control and Prevention. Chronic Fatigue Syndrome. May 26, 2006. Available at: www.cdc. gov/cfs. Accessed March 21, 2010.

5. Devanur LD, Kerr JR. Chronic fatigue syndrome. J Clin Virol 2006; 37: 139-50.

6. Jason LA, Taylor RR, Kennedy CL, et al. Chronic fatigue syndrome: Sociodemographic subtypes in a communitybased sample. Evaluation and the Health Professions 2000; 23: 243-63.

7. Torres-Harding SR, Jason LA, Taylor RR. Fatigue severity, attributions, medical utilization, and symptoms in persons with chronic fatigue. J Behav Med 2002; 25: 99-113.

8. Griffith JP, Jason LA, Porter N, et al. CFS: a review of epidemiology and natural history studies. Bulletin of the IACFS/ME 2009; 17: 96-8.

9. Fernández AA, Martín AP, Martínez MI, et al. Chronic fatigue syndrome: aetiology, diagnosis and treatment. BMC Psychiatry 2009; 9 (Suppl 1): S1.

10. Cope H, Mann A, Pelosi A, David A. Psychosocial risk factors for chronic fatigue and chronic fatigue syndrome following presumed viral illness: a case-control study. Psychol Med 1996; 26: 1197-209.

11. Dalpke AH, Reiner T, Ritter K. Oxidative injury to endothelial cells to Epstein-Barr virus-induced autoantibodies against superoxide dismutase. J Med Virol 2003; 71: 408-16.

12. Van Den Eede F, Moorkens G, Van Houdenhove B, Cosyns P, Claes SJ. Hypothalamic-pituitary-adrenal axis function in chronic fatigue syndrome. Neuropsychobiology 2007; 55: 112-20.

13. Cleare AJ. The neuroendocrinology of chronic fatigue syndrome. Endocrine Rev 2003; 24: 236-52.

14. Richards RS, Roberts TK, McGregor NR, Dunstan RH, Butt $\mathrm{HL}$. Blood parameters indicative of oxidative stress are associated with symptom expression in chronic fatigue syndrome. Redox Rep 2000; 5: 35-41.

15. Jammes Y, Steinberg JG, Mamabrini O, Bregeon F, Delliaux S. Chronic fatigue syndrome: assessment of increased oxidative stress and altered muscle excitability in response to incremental exercise. J Intern Med 2005; 257: 299-310.

16. Pall ML, Scatterle JD. Elevated nitric oxide/peroxynitrite mechanism for the common etiology of multiple chemical sensitivity, chronic fatigue syndrome, and posttraumatic stress disorder. Ann N Y Acad Sci 2001; 933: 323-9.

17. Logan AC, Wong C. Chronic fatigue syndrome: oxidative stress and dietary modifications. Altern Med Rev 2001; 6: 450-9.

18. Vecchiet J, Cipollone F, Falasca K, et al. Relationship between musculoskeletal symptoms and blood markers 
of oxidative stress in patients with chronic fatigue syndrome. Neurosci Lett 2003; 335: 151-4.

19. Kennedy G, Spence, VA, McLaren M, Hill A, Underwood C, Belch J. Oxidative stress levels are raised in chronic fatigue syndrome and are associated with clinical symptoms. Free Rad Bio Med 2005; 39: 584-9.

20. Fulle S, Mecocci P, Fano G, et al. Specific oxidative alterations in vastus lateralis muscle of patients with the diagnosis of chronic fatigue syndrome. Free Radic Biol Med 2000; 29: 1252-9.

21. Keenoy MY, Moorkens G, Vertommen J, De Leeuw I. Antioxidant status and lipoprotein peroxidation in chronic fatigue syndrome. Life Sci 2001; 68: 2037-49.

22. Del Rio D, Stewart A, Pellegrini N. A review of recent studies on malondialdehyde as toxic molecule and biological marker of oxidative stress. Nutr Metab Cardiovasc Dis 2005; 15: 316-28.

23. Polidori MC, Savino K, Alunni G, et al. Plasma lipophilic antioxidants and malondialdehyde in congestive heart failure patients: relationship to disease severity. Free Radic Biol Med 2002; 32: 148-52.

24. Loguercio C, Federico A. Oxidative stress in viral and alcoholic hepatitis. Free Radic Biol Med 2003; 34: 1-10.

25. Delibas N, Ozcankaya R, Altuntas I. Clinical importance of erythrocyte malondialdehyde levels as a marker for cognitive deterioration in patients with dementia of Alzheimer type: a repeated study in 5-year interval. Clin Biochem 2002; 35: 137-41.

26. VanderVeen LA, Hashim MF, Shyr Y, Marnett LJ. Induction of frame shift and base pair substitution mutations by the major DNA adduct of the endogenous carcinogen malondialdehyde. Proc Natl Acad Sci U S A 2003; 100: 14247-52.

27. Niedernhofer LJ, Daniels JS, Rouzer CA, Greene RE, Marnett LJ. Malondialdehyde, a product of lipid peroxidation, is mutagenic in human cells. J Biol Chem 2003; 278: 31426-33.

28. Sakuma S, Sumi H, Kohda T, Arakawa Y, Fujimoto Y. Effects of lipid peroxidation-derived products on the growth of human colorectal cancer cell line HT-29. J Clin Biochem Nutr 2009; 45: 171-7.

29. Catalá A. Lipid peroxidation of membrane phospholipids generates hydroxy-alkenals and oxidized phospholipids active in physiological and/or pathological conditions. Chem Phys Lipids 2009; 157: 1-11.

30. Smirnova IV, Pall ML. Elevated levels of protein carbonyls in sera of chronic fatigue syndrome patients. Mol Cell Biochem 2003; 248: 93-5.

31. Beal MF. Oxidatively modified proteins in aging and disease. Free Radic Biol Med 2002; 32: 797-803.

32. Dalle-Donne I, Rossi R, Giustarini D, Milzania A, Colombo R. Protein carbonyl groups as biomarkers of oxidative stress. Clinica Chimica Acta 2003; 329: 23-38.

33. Dalle-Donne I, Giustarini D, Colombo R, Rossi R, Milzani A. Protein carbonylation in human diseases. Trends Mol Med 2003; 9: 169-76.

34. Aaronson LS, Teel SC, Cassmeyer V, et al. Definiting and measuring fatigue J Nursing Scholarhip 1999; 31: 1.

35. Zachisson O, Regland B, Jahreskog M, Kron M, Gotfries C. A rating scale for fibromyalgia and chronic fatigue syndrome (the Fibro Fatigue Scale). J Psychosom Res 2002; 52: 501-9.

36. Garcia-Campayo J, Pascual A, Aida M, Marzo J, Magallon R, Fortes S. The Spanish version of the Fibro Fatigue Scale:validation of a questionnaire for the observers assessment of fibromyalgia and chronic fatigue syndrom. Gen Hosp Psych 2006; 28: 154-60.

37. Uchida K. Role of reactive aldehyde in cardiovascular diseases. Free Radic Biol Med 2000; 28: 1685-96.
38. Tamer L, Sucu N, Polat G, et al. Decreased serum total antioxidant status and erythrocyte-reduced glutathione levels are associated with increased serummalondialdehyde in atherosclerotic patients. Arch Med Res 2002; 33: 257-60.

39. Boaz M, Matas Z, Biro A, et al. Serum malondialdehyde and prevalent cardiovascular disease in hemodialysis. Kidney Int 1999; 56: 1078-83.

40. Boaz M, Matas Z, Biro A, et al. Comparison of hemostatic factors and serum malondialdehyde as predictive factors for cardiovascular disease in hemodialysis patients. Am J Kidney Dis 1999; 34: 438-44.

41. Chevion M, Berenshtein E, Stadtman ER. Human studies related to protein oxidation: protein carbonyl content as a marker of damage. Free Radic Res 2000; 33: S99-108.

42. Shacter E. Quantification and significance of protein oxidation in biological samples. Drug Metab Rev 2000; 32: 307-26.

43. Dodani S, Marion L, Sharma G, Reed GL, George V. The association of carotid intima media thickness with dysfunctional HDL in South Asians. Arch Med Sci 2008; 4: 40-6.

44. Vulic D, Loncar S, Krneta M, et al. Risk factor control and adherence to treatment in patients with coronary heart disease in the Republic of Srpska, Bosnia and Herzegovina in 2005-2006. Arch Med Sci 2010; 6: 270-5.

45. Mark L, Paragh G, Karadi I, Reiber I, Pados G. Changes in attainment of lipid goals by general practitioners and specialists in patients at high cardiovascular risk in Hungary during 2004-2008. Arch Med Sci 2010; 6: 695-700.

46. Macphee CH, Nelson JJ, Zalewski A. Lipoprotein associated phospholipase A2 as a target of therapy. Curr Opin Lipidol 2005; 16: 442-6.

47. Barter PJ, Nicholls S, Rye KA, Anantharamaiah GM, Navab $M$, Fogelman AM. Antiinflammatory properties of HDL. Circ Res 2004; 95: 764-72.

48. Kędziora-Kornatowska K, Czuczejko J, Motyl J, et al. Effects of coenzyme Q10 supplementation on activities of selected antioxidative enzymes and lipid peroxidation in hypertensive patients treated with indapamide. Arch Med Sci 2010; 6: 513-8.

49. Aldred S, Bennett S, Mecocci P. Increased low-density lipoprotein oxidation, but not total plasma protein oxidation, in Alzheimer's disease. Clin Biochem 2010; 21: 267-71.

50. Kostulski A, Pawełczyk T, Rabe-Jabłońska J. The risk of significant body weight gain and abdominal obesity during short-term treatment with olanzapine Arch Med Sci 2009; 5: 259-66.

51. Spence VA, Kennedy G, Belch JJF, Hill A, Khan F. Low-grade inflammation and arterial wave reflection in patients with chronic fatigue syndrome. Clin Sci 2008; 114: 561-6. 